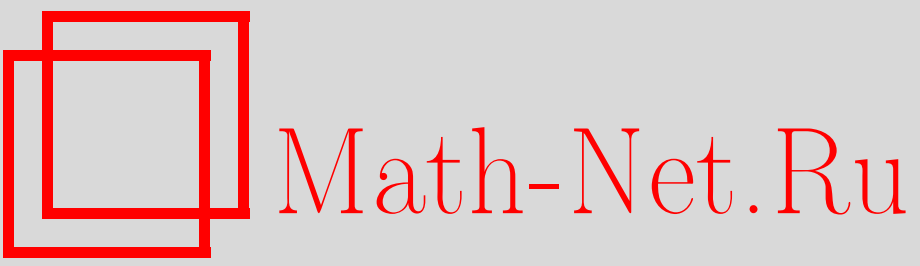

А. Б. Поляков, О равновесных распределениях на множестве перестановок целых чисел, УМН, 1999, том 54, выпуск 2, 183-184

DOI: https://doi.org/10.4213/rm145

Использование Общероссийского математического портала Math-Net.Ru подразумевает, что вы прочитали и согласны с пользовательским соглашением

http://www . mathnet.ru/rus/agreement

Параметры загрузки:

IP: 107.22 .136 .117

26 апреля 2023 г., 15:36:53 


\title{
О РАВНОВЕСНЫХ РАСПРЕДЕЛЕНИЯХ НА МНОЖЕСТВЕ ПЕРЕСТАНОВОК ЦЕЛЫХ ЧИСЕЛ
}

\author{
А. Б. Поляков
}

1. В связи с некоторыми вопросами квантовой статистической механики, К.-Х. Фихтнер рассмотрел в [1] специальный класс случайных перестановок счетного множества. Вкратце опишем его модель, поскольку она существенно используется в этой заметке.

Пусть $\mathscr{Z}$-произвольное счетное множество. Для любых $Z_{1}, Z_{2} \subset \mathscr{Z}$ обозначим через $E_{Z_{1}, Z_{2}}$ множество всех биективных отображений из $Z_{1}$ в $Z_{2}$.

Пусть $\alpha=\left(\alpha_{x, y}\right), x, y \in \mathscr{Z},-$ матрица, удовлетворяющая условиям:

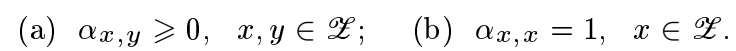

Для всякого конечного подмножества $Z^{\prime} \subset \mathscr{Z}$ определим вероятностную меру $P_{Z^{\prime}}$ на множестве $\left(Z^{\prime}\right)^{Z^{\prime}}=\left\{g: Z^{\prime} \rightarrow Z^{\prime}\right\}$ по формуле: $P_{Z^{\prime}}(\{g\})=0$, если $g \notin E_{Z^{\prime}, Z^{\prime}}$ и и

$$
P_{Z^{\prime}}(\{g\})=\prod_{x \in Z^{\prime}} \alpha_{x, g(x)} / \sum_{h \in E_{Z^{\prime}, Z^{\prime}}} \prod_{x \in Z^{\prime}} \alpha_{x, h(x)} \text {, если } g \in E_{Z^{\prime}, Z^{\prime}} .
$$

На множестве $\mathscr{Z}^{\mathscr{Z}}=\{f: \mathscr{Z} \rightarrow \mathscr{Z}\}$ введем цилиндрическую $\sigma$-алгебру $\mathscr{F}(\mathscr{Z})$. Пусть $E_{\mathscr{Z}}$ - множество перестановок $g \in E_{\mathscr{Z}, \mathscr{L}}$, разлагающихся на конечные циклы. Потребуем, чтобы матрица $\alpha$ удовлетворяла условию "компактности":

$$
\text { (c) } \sum_{A \subset \mathscr{Z},|A|<\infty, y \in A} \sum_{g \in D_{A}} \prod_{x \in A} \alpha_{x, g(x)}<\infty, \quad y \in \mathscr{Z} \text {, }
$$

где $D_{A}$ - совокупность всех циклических перестановок множества $A$.

ТеОрема 1 [1]. Если выполнены условия (а)-(c), то для всякой последовательности $\left\{Z_{l}\right\}_{l=1}^{\infty}$ конечных подмножеств $\mathscr{Z}, Z_{l} \subset Z_{l+1}, \bigcup_{l} Z_{l}=\mathscr{Z}$, существуют подпоследовательность $\left\{Z_{l(n)}\right\}_{n=1}^{\infty}$ и вероятностная мера $P$ на $\left(\mathscr{Z}^{\mathscr{Z}}, \mathscr{F}(\mathscr{Z})\right)$ такие, что 1) $P=$ $\lim _{n \rightarrow \infty} P_{Z_{l(n)}}$ в смысле сходимости всех конечномерных распределений; 2) $P\left(E_{\mathscr{Z}}\right)=1$.

2. Рассмотрим в качестве $\mathscr{Z}$ одномерную целочисленную решетку $\mathbb{Z}$, положим $\Omega=\mathbb{Z}^{\mathbb{Z}}$, $\mathscr{F}=\mathscr{F}(\mathbb{Z})$ и зададим преобразование $T: \Omega \rightarrow \Omega$ по формуле:

$$
T(f)(x)=f(x+1)-1, \quad x \in \mathbb{Z}, \quad f \in \Omega .
$$

Очевидно, $T$ - взаимнооднозначное отображение, $T$ и $T^{-1}$ измеримы и $T E_{\mathbb{Z}}=E_{\mathbb{Z}}$.

Цель этой заметки - ввести на множестве перестановок $E_{\mathbb{Z}}$ класс $T$-инвариантных мер, которые в известном смысле можно считать равновесными состояниями. Для этого будем рассматривать теплицевы матрицы $\alpha=\left(\alpha_{x, y}\right), x, y \in \mathbb{Z}: \alpha_{x, y}=\alpha_{x^{\prime}, y^{\prime}}$, если $y-x=y^{\prime}-x^{\prime}$. Положим $\alpha_{j}=\alpha_{x, y}$, когда $y-x=j, j \in \mathbb{Z}$, и $S(\alpha)=\left\{j \in \mathbb{Z}: \alpha_{j} \neq 0\right\}$.

Пусть вначале множество $S(\alpha)$ конечно. Положим $k(\alpha)=\max \{|j|: j \in S(\alpha)\}$, если $\max \{|j|:$ $j \in S(\alpha)\} \neq 0$, и $k(\alpha)=1$ в противном случае.

ОПредЕлЕнИЕ. Пусть $S \subset \mathbb{Z}$. Последовательность $\left(a_{1}, \ldots, a_{m}\right), a_{i} \in S$, назовем $S$-допустимой, если существуют $n \in \mathbb{N}, n \geqslant m$, и перестановка $g$ множества $N_{n}=\{1,2, \ldots, n\}$ такие, что: 1) $\left.g(i)-i \in S, i \in N_{n} ; 2\right)$ существует подмножество $\left\{i_{0}, i_{0}+1, \ldots, i_{0}+m-1\right\} \subset N_{n}$ такое, что $g\left(i_{0}+l\right)-i_{0}-l=a_{l+1}, l=0,1, \ldots, m-1$.

Построим ориентированный конечньй связный граф $\Gamma(\alpha)$, взяв в качестве его вершин все $S(\alpha)$-допустимые последовательности длины $2 k, k=k(\alpha)$, а в качестве ребер такие пары вершин $\bar{a}=\left(a_{1}, \ldots, a_{2 k}\right), \bar{a}^{\prime}=\left(a_{1}^{\prime}, \ldots, a_{2 k}^{\prime}\right)$, что 1) $a_{2}=a_{1}^{\prime}, a_{3}=a_{2}^{\prime}, \ldots, a_{2 k}=a_{2 k-1}^{\prime}$; 2) $\left(a_{1}, \ldots, a_{2 k}, a_{2 k}^{\prime}\right) \quad S(\alpha)$-допустима. Для любых двух вершин $\bar{a}=\left(a_{1}, \ldots, a_{2 k}\right)$ и $\bar{a}^{\prime}=$ $\left(a_{1}^{\prime}, \ldots, a_{2 k}^{\prime}\right)$ положим $A_{\bar{a} \bar{a}^{\prime}}(\alpha)=\alpha_{a_{2 k}^{\prime}}$, если $\left(\bar{a}, \bar{a}^{\prime}\right)$ - ребро в $\Gamma(\alpha)$, и $A_{\bar{a} \bar{a}^{\prime}}(\alpha)=0$ в противном случае. Далее, введем матрицу $A(\alpha) \stackrel{2 k}{=}\left(A_{\bar{a} \bar{a}^{\prime}}(\alpha)\right)$, обозначим $Y(\Gamma(\alpha))$ множество бесконечных в обе стороны путей в $\Gamma(\alpha)$ и положим $E_{\mathbb{Z}}(\alpha)=\left\{g \in E_{\mathbb{Z}}: g(x)-x \in S(\alpha), x \in \mathbb{Z}\right\}$. 
Теорема 2. Пусть $\alpha=\left(\alpha_{x}, y\right)$ - теплицева матрица, удовлетворяющая условиям (а) $-($ c) $и|S(\alpha)|<\infty ; k=k(\alpha)$. Тогда существует $T$-инвариантная вероятностная мера $P$ такая, что $P\left(E_{\mathbb{Z}}(\alpha)\right)=1$ и $P=\lim _{l \rightarrow \infty} P_{Z}$ для всякой последовательности $\left\{Z_{l}\right\}_{l=1}^{\infty}$ конечных подмножеств $\mathbb{Z}, Z_{l} \subset Z_{l+1}$ u $\bigcup_{l} Z_{l}=\mathbb{Z} ;$ динамическая система $\left(E_{\mathbb{Z}}(\alpha), P, T\right)$ изоморфна системе $(Y(\Gamma(\alpha)), \widetilde{P}, \widetilde{T})$, где $\widetilde{T}$ - сдвиг на один шаг влево на $Y(\Gamma(\alpha))$, а $\widetilde{P}-$ равновесная марковская мера [2] для матрицы $A(\alpha)$.

3. Обратимся к теплищевым матрицам $\left(\alpha_{x, y}\right)$ с $|S(\alpha)|=\infty$. Ограничимся при этом симметрическими матрицами $\left(\alpha_{j}=\alpha_{-j}, j \in \mathbb{Z}\right)$. По $\alpha=\left(\alpha_{x, y}\right)$ определим теплицеву матрицу $\alpha^{(m)}=\left(\alpha_{x, y}^{m}\right), m \geqslant 1$, положив $\alpha_{x, y}^{m}=\alpha_{x, y}$, если $|y-x| \leqslant m$, и $\alpha_{x, y}^{m}=0$, если $|y-x|>m$.

Согласно теореме 2 , для $\alpha^{(m)}$ определена вероятностная мера $P=P_{\alpha}^{(m)}, m \geqslant 1$. Рассмотрим последовательность чисел $\gamma_{1}, \ldots, \gamma_{l}$ таких, что $0 \leqslant \gamma_{i} \leqslant q^{i}$, где $q \in(0,1 / 3), 1 \leqslant i \leqslant l$, и последовательность натуральных чисел $\left\{j_{r}\right\}_{l+1}^{\infty}, l<j_{r}, j_{r}<j_{r+1}$.

ТеОрема 3. Для любых $\gamma_{1}, \ldots, \gamma_{l} u\left\{j_{r}\right\}_{l+1}^{\infty}$, указанных выше, существует последовательность положительных чисел $\left\{\eta_{r}\right\}_{l+1}^{\infty}$ такая, что для всякой симметрической тепличевой матрищь $\alpha$ c $|S(\alpha)|=\infty$, удовлетворяющей условиям (a)-(c) и следующим ограничениям: 1) $\alpha_{j}=\gamma_{j}, 1 \leqslant j \leqslant l$; 2) $\left.0<\alpha_{j r} \leqslant \eta_{r}, r=l+1, l+2, \ldots ; 3\right) \alpha_{j}=0$, если $j>l u j \neq j_{r}, r=l+1, l+2, \ldots$, последовательность вероятностных мер $\left\{P_{\alpha}^{(m)}\right\}_{m=1}^{\infty}$ слабо сходится к некоторой вероятностной мере $P_{\alpha}$ на $(\Omega, \mathscr{F})$.

Теорема 4. Если выполнены условия теоремы 3, то

а) мера $P_{\alpha} T$-инвариантна; б) $P_{\alpha}\left(E_{\mathbb{Z}}(\alpha)\right)=1$; в) $P_{\alpha}$ есть гиббсовское распределение для матрицы $\alpha$ в следующем смьсле [1]: для всякого конечного множества $\Lambda \subset \mathbb{Z} u$ любого $g_{1}: \Lambda \rightarrow \mathbb{Z}$ при $P_{\alpha}$-n.в. $f \in \Omega$

$$
\begin{aligned}
P_{\alpha}(\{g \in \Omega & \left.\left.: g(x)=g_{1}(x), x \in \Lambda\right\} \mid \mathscr{F}_{\mathbb{Z} \backslash \Lambda}\right)(f) \\
& = \begin{cases}\prod_{x \in \Lambda} \alpha_{x, g_{1}(x)} / \sum_{h \in E_{\Lambda, g_{1}(\Lambda)}} \prod_{x \in \Lambda} \alpha_{x, h(x)}, & \text { если } f(\mathbb{Z} \backslash \Lambda)=\mathbb{Z} \backslash g_{1}(\Lambda), \\
0 & \text { в противном случае. }\end{cases}
\end{aligned}
$$

Обозначим через $\mathscr{M}(\alpha, T)$ множество всех $T$-инвариантных вероятностных мер $P$ на $(\Omega, \mathscr{F})$ с конечной энтропией $h_{P}(T)<\infty$ и таких, что $P\left(E_{\mathbb{Z}}(\alpha)\right)=1$. Определим функцию $v_{\alpha}: E_{\mathbb{Z}}(\alpha) \rightarrow \mathbb{R}$ по формуле $v_{\alpha}(g)=\ln \alpha_{g(0)}$. Обозначим $\lambda_{m}(\alpha)$ максимальное собственное значение матрицы $A\left(\alpha^{(m)}\right)$ и $\lambda(\alpha)=\lim _{m \rightarrow \infty} \lambda_{m}(\alpha)$.

ТеОРема 5. Для всякой последовательности натуральных чисел $\left\{j_{r}\right\}_{r=1}^{\infty}, 1<$ $j_{1}<\cdots<j_{r}<\cdots$, существует последовательность положительных чисел $\left\{\eta_{r}\right\}_{r=1}^{\infty}$, $\eta_{r} \rightarrow 0$, такая, что если симметрическая теплицева матрица $\alpha$ c $|S(\alpha)|=\infty$ удовлетворяет условиям (a)-(c) $u$, кроме того, 1) $\left.0<\alpha_{1} \leqslant 1 / 3 ; 2\right) \alpha_{j}=0$, если $\left.1<j \neq j_{r}, \quad r \geqslant 1 ; 3\right) \quad 0<\alpha_{j_{r+1}}<\alpha_{j_{r}}<\eta_{r}, \quad r \geqslant 1 ; \quad$ mo a) $\lambda(\alpha)<\infty$ $u \sup _{P \in \mathscr{M}(\alpha, T)}\left\{h_{P}(T)+\int_{E_{\mathbb{Z}}(\alpha)} v_{\alpha}(g) d P(g)\right\}=\ln \lambda(\alpha) ;$ б) мера $P_{\alpha}$ принадлежст $\mathscr{M}(\alpha, T)$ иявляется равновесным состоянием, т.е. на ней достигается верхняя грань функционала в а).

Автор выражает благодарность Б. М. Гуревичу за постановку задачи.

\section{СПИСОК ЛИТЕРАТУРЫ}

[1] Fichtner K. H. // Probab. Theory Related Fields. 1991. V. 89. № 1. P. 35-60. [2] Гeopги Х.-О. Гиббсовские меры и фазовые переходы. М.: Мир, 1992. 